the whole of which is devoted to astronomy as related to cosmology. The fundamental discovery of the two stellar populations has shed light on the evolutionary history of a galaxy, and this has been reinforced by studies of the origin of the chemical elements and of the nuclear processes which take place in stars. Astronomers are now"aware of the basic nature of interstellar material as the source of the bright stars of Population I, and when a collision of galaxies occurs the interstellar matter which they contrin is the seat of the optical and radio emissions which are observed. A decision between evolutionary and steady-state cosmologies is much to be desired; measurement of the red-shifts of more distant nebulæ, identification of additional distant colliding galaxies as radio sources, further investigations on the large-scale distribution of galaxies, all may contribute to the decision. Observational programmes now in progress may indeed provide the essential data for the solution of the cosmological problem.

\section{Occultations of Radio Sources by the Moon}

A PAPER, by F. Link, entitled "Occultations des Radiosources par la Lune et Phénomènes Connexes" (Bull. Astro. Instit. Czechoslovakia, 7, No. 1 ; 1956), deals with the possibility of occultations by the Moon of radio sources and shows the existence of a very rarified lunar atmosphere. In an earlier publication $(5,112 ; 1954)$ Link directed attention to a series of such occultations between the years 1955 and 1957 , and C. L. Seeger has also directed attention to the same subject (Trans. Int. Astro. Union, 8 ; 1952). The investigation has more than an academic interest, and Link refers to four matters which are of great interest and importance : the exact determination of the positions of radio sources ; the determination of the photometric profile of radio sources; the discovery of new feeble radio sources; and possible proofs of the lunar ionosphere. As the last-mentioned can modify the interpretation of the first three points, it is dealt with first in the paper. Link points out that different attempts based on the optical properties of the lunar atmosphere have not, so far led to definite results. Lyot and Dollfus, by measuring with the coronagraph the brightness in the vicinity of the Moon's horns at the first quarter, estimated the superior limit of the density relative to that of the Earth's atmosphere at sea-level as $10^{-8}$ approximately. Dollfus, continuing with the investigations and basing his research on the polarization of the light diffused by the hypothetical atmosphere of the Moon, obtained a limit of about $10^{-8}$, while Kuiper, using the spectroscopic method to detect traces of sulphur dioxide, gave the mass of a vertical column of this gas the upper limit $3 \times 10^{-10}$ that of the terrestrial atmosphere. Other attempts by Lyot and Dollfus by the method previously described did not give such low limits. Link investigates the subject, starting with the two factors that determine the existence of any ionosphere-terrestrial or lunar ; these are the presence of gases capable of being ionized, and the ionizing solar radiation. Examining these two factors on the Earth and on the Moon, his mathematical investigations lead to the suggestion that a lunar atmosphere of density $10^{-12}$ that of the terrestrial atmosphere at sea-level might give observable phenomena such as the deviation of the rays and the augmentation of their intensity. It is admitted at the end of the paper that it is premature at present to pursue these general considerations, their aim being merely to direct attention to occulta. tions of radio sources and the phenomena which ean accompany them.

\section{Water Resources of Great Britain}

THE problem of water conservation and flood control in Britain is receiving increasing attention by the authorities concerned (see Nature, 176, 1133 ; 1955) and, in particular, has become more widely recognized during the present year as a result of the contrasts of water shortage during a dry winter and much recurrent flooding in the course of a wet summer. The publication of the "Surface Water Year-Book of Great Britain, 195354" (pp. 117. London : H.M.S.O., 1956; $30 s$. net) is a welcome reminder of the work now in hand to assess the water resources of the country on a more scientific basis. This publication of the Ministry of Housing and Local Government and of the Scottish Office gives hydrographic statistics for British rivers, together with related rainfalls, for the year ended September 30, 1954, and is the first of the intended yearly issues covering periods of one water year. The two previous publications embraced eight years each, and the records were somewhat patchy and broken. The new annual issue will, it is hoped, ensure both uniformity and continuity. The new volume contains the flow records of a hundred and two stations, has the daily discharges of most, and includes discharge frequencies for certain selected stations. A new feature is the inclusion of natural water temperatures of the River Test in Hampshire and the River Avon in Somerset. It is hoped to extend these observations into a network of temperature-recording stations which will eventually provide standards of comparison to which intermediate observations, in place or time, may be related.

\section{Standard Capacitors and their Accuracy in Practice}

No. 13 in the series of "Notes on Applied Science" of the National Physical Laboratory, Teddington, which is on "Standard Capacitors and their Accuracy in Practice" (pp. 10. London: H.M.S.O., 1955; 1s. $3 d$. net), directs attention to the various factors which affect the performance of capacitors, both by virtue of their construction and by their method of use. The booklet is primarily concerned with capacitors designed for use as laboratory standards, and the information given is based on the wide experience gained in testing and using such instruments over many years at the Laboratory. Both air-dielectric and solid-dielectric capacitors are discussed, and separate sections deal with the inductance and resistance of capacitors and with the effects of leads on the measured values of the capacitances. Goodquality capacitors, whether with air or mica dielectric, it is concluded, provide a standard of reactance not greatly inferior in constancy to that of a good resistor, provided that proper precautions are taken in their maintenance and use. Clamped mica capacitors of between 0.1 and $1 \mu \mathrm{F}$. are probably the most stable, and if they are kept under moderate and uniform conditions of temperature and humidity the fluctua. tions about their mean values should not be more than \pm 0.5 part in $10^{4}$ on capacitance and \pm 20 per cent on power factor, over a period of several years. The capacitance of a capacitor of simple geometric shape can be calculated from its linear dimensions and from the dielectric constant of the material be. 